\title{
DYNAMIC COUPLING OF MULTI-PHASE FLUIDS WITH A MOVING OBSTACLE
}

Tso-Ren Wu

Graduate Institute of Hydrological and Oceanic Sciences, National Central University, Jhongli City, Taoyuan County, Taiwan, R.O.C., tsoren@ncu.edu.tw

Chih-Jung Huang

Department of Civil Engineering, National Central University, Jhongli City, Taoyuan County, Taiwan, R.O.C.

Mei-Hui Chuang

Graduate Institute of Hydrological and Oceanic Sciences, National Central University, Jhongli City, Taoyuan County, Taiwan, R.O.C.

Chung-Yue Wang

Department of Civil Engineering, National Central University, Jhongli City, Taoyuan County, Taiwan, R.O.C.

Chia-Ren Chu

Department of Civil Engineering, National Central University, Jhongli City, Taoyuan County, Taiwan, R.O.C.

Follow this and additional works at: https://jmstt.ntou.edu.tw/journal

Part of the Engineering Commons

\section{Recommended Citation}

Wu, Tso-Ren; Huang, Chih-Jung; Chuang, Mei-Hui; Wang, Chung-Yue; and Chu, Chia-Ren (2011) "DYNAMIC COUPLING OF MULTI-PHASE FLUIDS WITH A MOVING OBSTACLE," Journal of Marine Science and Technology. Vol. 19: Iss. 6, Article 8.

DOI: $10.51400 / 2709-6998.2206$

Available at: https://jmstt.ntou.edu.tw/journal/vol19/iss6/8

This Research Article is brought to you for free and open access by Journal of Marine Science and Technology. It has been accepted for inclusion in Journal of Marine Science and Technology by an authorized editor of Journal of Marine Science and Technology. 


\section{DYNAMIC COUPLING OF MULTI-PHASE FLUIDS WITH A MOVING OBSTACLE}

\section{Acknowledgements}

The authors are grateful to National Science Council, Taiwan, for the financial support under the Grant NSC 95-2116- M-008-006-MY3, with TEC Contribution Number 00040. 


\title{
DYNAMIC COUPLING OF MULTI-PHASE FLUIDS WITH A MOVING OBSTACLE
}

\author{
Tso-Ren $\mathrm{Wu}^{*}$, Chih-Jung Huang**, Mei-Hui Chuang*, \\ Chung-Yue Wang**, and Chia-Ren Chu**
}

Key words: moving solid algorithm, discrete element method (DEM), volume of fluid (VOF), floating cube, landslide tsunami.

\begin{abstract}
In this paper, we present a two-way coupled moving solid algorithm with discrete element method (DEM) to simulate dynamic fluid-solid interaction. This model allows obstacles moving in the multi-phase fluids to generate realistic motion for both the fluids and obstacles. We discretize the NavierStokes equation using finite volume method (FVM) for the fluid part. The volume-of-fluid (VOF) method is used to track the fluid-fluid and fluid-solid interfaces. This algorithm is useful in the fields of Engineering and Earth Sciences, such as structure collapse and landslide generated tsunamis. Examples of landslide tsunami and floating cubes are demonstrated using the algorithm.
\end{abstract}

\section{INTRODUCTION}

The numerical simulation of water waves interacting with a moving solid body is important for many sciences and engineering applications, such as landslide generated tsunamis, caisson work, ship maneuvering, and wave energy. However, to analyze unsteady flows with free surfaces poses a great challenge to numerical simulations, because both the freesurface boundary and the moving solid boundary are presented in the fluid system, and both elements are parts of the solutions.

Over the past decay, many numerical schemes solved the Navier-Stokes (NS) equations have been developed for simulation of wave-structure interactions. Lin and $\mathrm{Li}$ [15] made use of $\sigma$-coordinate transformation to map an irregular physical domain on a rectangular computational grid. Lin [14] further developed a 3D multiple-layer $\sigma$-coordinate model to

Paper submitted 02/25/10; revised 07/14/11; accepted 11/17/11. Author for correspondence: Tso-RenWu (e-mail: tsoren@ncu.edu.tw).

*Graduate Institute of Hydrological and Oceanic Sciences, National Central University, Jhongli City, Taoyuan County, Taiwan, R.O.C.

**Department of Civil Engineering, National Central University, Jhongli City, Taoyuan County, Taiwan, R.O.C. simulate surface wave interaction with structures. However, the $\sigma$-coordinate method requires coordinate transformation which is hard to implement in 3D breaking waves and expensive. A similar problem happens to ALE (arbitrary LagrangianEulerian) method [11]. In ALE method, domain re-meshing is required if the solid obstacle is moving. The re-meshing process is costly and difficult to implement in $3 \mathrm{D}$ with complex geometry.

Peskin [21] developed the immersed boundary method (IB method) $[2,6,12,17,18]$. The fixed-grid technique constructed on either simple Cartesian coordinates or complex unstructured girds is adopted to handle moving bodies and complex breaking waves and is divided into two categories, depending on how the inertial solid boundaries are treated. Shen and Chan [23] combined IB method and VOF to study the fluid interaction with submerged structures. In IB method, both the solid and fluid are treated as one fluid, and a fictitious force is applied to the solid domain. This method is also known as the fictitious domain method [7,8]. The one-fluid assumption tends to have a larger error when fluid-fluid interfaces appears in the computational domain. A similar problem occurs in the penalty method $[3,22]$. In the penalty method, the zero-shear constrain is applied to the solid domain. However, the one-fluid assumption limits the accuracy when the solid obstacle is moving between the air-water interface.

Alternative to the one-fluid assumption, we solve the fluid and solid separately. This approach is based on the Partialcell (PC) method. The PC (sometimes also called "cut-cell" or "CC") method has been widely employed to represent arbitrary body configuration. Combined with the VOF freesurface treatment $[10,13]$, PC method has been developed for the modeling of complex breaking waves interacting with stationary solid boundaries. Heinrich [9] solved 2D NS equations and added a source function to the continuity and momentum equations to represent moving boundary effects. $\mathrm{Wu}$ [30] and Liu et al. [16] further extended Heinrich's model to simulate the $3 \mathrm{D}$ wave generation induced by landslides. In their studies, the movements of the obstacles are prescribed, while we are more interested in the "two-way" fluid-solid coupling which requires the prediction of the solid-body movement. 
The body motion with large translation and rotation can be calculated by some computational methods developed in solid mechanics, like the discrete element method (DEM) [4, 5], discontinuous deformation analysis (DDA) method [3], and Manifold method (MM) [24, 26], vector form intrinsic finite element (VFIFE) method [25, 27] and some other nonlinear finite element analysis methods based on up-dated Lagrangian algorithm [28]. For the system containing discrete bodies, the contact detection and contact force analysis are the core parts of the motion analysis. Cundall [1] proposed the "common plane" concept to do the contact detection among threedimensional polyhedrons. Recently, Zhao et al. [31] improved the common plane algorithm to accelerate the contact detection speed of granular particles of irregular shapes. Wang et al. [29] developed a directed contact analysis algorithm for the motion analysis of mixed polyhedral and ellipsoidal particles. For the contact analysis of deformable bodies, Zhong and Nilsson [32] had developed an effective contact searching algorithm for general 3-D contact impact problems. The authors of this paper would like to group these methods by the name as "discrete body motion analysis method".

To include the fluid effect into the analysis of granular medium is highly interested by the researchers in the past two decades. Muller et al. [19, 20] used the smooth particle hydrodynamics (SPH) to model the free fluid surface and conducted the fluid-solid particle interaction analysis based on the Eulerian grid-based mode. However, error is accumulated after some interactions among particles, especially for the particles of distinct types.

In this paper, we propose a new method which couples the discrete body motion analysis method and a moving-solid algorithm based on the PC-VOF method. Our method allows robust and accurate solid-fluid coupling in either structure or on unstructured girds. The body motion can either be prescribed or based on DEM. The fluid solver adopts the twostep projection for solving 3D NS equations for fluid-solid interaction by simultaneously conserving the momentum of the fluid and the moving body, while enforcing the conservation of fluid mass. For the model implementations, we will demonstrate and validate the results by landslide tsunamis and floating cubes. Good agreement of the obstacle trajectories of the landslide experiments and numerical prediction can be seen.

\section{NUMERICAL MODEL}

In this section, the numerical algorithm used to simulate fluid motion is introduced. The model uses VOF method for the free-surface tracking, finite volume method for the discretization, PC method for placing the solid material inside the domain, and moving solid algorithm for simulating the fluid motion caused by a moving solid body. The motion of solid is estimated by DEM model.

In the projection method, the momentum of Navier-Stokes equations can be described by two fractional steps:

$$
\begin{aligned}
\frac{\rho^{n+1} \mathbf{u}^{*}-\rho^{n} \mathbf{u}^{n}}{\Delta t}= & -\nabla \cdot(\rho \mathbf{u u})^{n}-\nabla P^{n} \\
& +\nabla \cdot\left(\mu^{n}\left(\nabla \mathbf{u}+\nabla^{T} \mathbf{u}\right)^{n}\right)+\rho^{n} \mathbf{g}^{n} \\
\frac{\rho^{n+1} \mathbf{u}^{n+1}-\rho^{n+1} \mathbf{u}^{*}}{\Delta t} & =-\nabla \delta P^{n+1}+\rho^{n+1} \mathbf{g}^{n+1}-\rho^{n} \mathbf{g}^{n}
\end{aligned}
$$

where $\rho$ is the fluid density, $P$ is the total pressure, $\mathbf{g}$ is gravity, and $\mu$ is viscosity. The superscripts $n$ and $n+1$ represent the current time and next time, respectively. The superscript $*$ represents an intermediate level. From combining Eqs. (1) and (2), we can get Poisson Pressure Equation (PPE) to solve the next time-step pressure $P^{n+1}$ :

$$
\nabla \cdot \frac{\nabla \delta P^{n+1}}{\rho^{n+1}}=\nabla \cdot\left(\frac{\mathbf{u}^{*}}{\Delta t}\right)
$$

\section{Volume of Fraction Method for Multi-Phase Fluids}

In order to simulate multi-fluids with interfaces, such as air and water, the VOF method is adopted. A volume of fluid fraction, $f$ representing the volume fraction of water is defended the ratio of the fluid to total volumes. If the cell is full of water, the value of $f$ is equal to 1 ; if the cell is empty, the value is zero; if the value is $0<f<1$, it means the cell contains air-water interface. A set of mass and momentum conservation equations is solved on a fixed grid. The mass conservation law can be transformed to VOF equation:

$$
\frac{\partial f}{\partial t}+\nabla \cdot(f \mathbf{u})=0
$$

where $\mathbf{u}$ is the velocity field, and $f$ is volume of fluid fraction.

From PLIC (piecewise linear interface calculation) algorithm, the interface between air and water can be described.

$$
\frac{f^{n+1}-f^{n}}{d t}=-\mathbf{u}^{n} \cdot \nabla f^{n}
$$

\section{Finite Volume Method and Partial-Cell Method}

The fluid flow fields can be discretized by Finite Volume Method (FVM). In a control volume, the arbitrary quantity $\phi$ in governing equation can be defined:

$$
\frac{\partial \phi}{\partial t}+\nabla \cdot\left(u_{i} \phi\right)=S(\phi)
$$

where $S$ is a source term which is related to $\phi$. By applying integral and Gauss Divergence theorem in the finite volume, a discrete numerical scheme is presented: 


$$
\frac{\phi_{i}^{n+1}-\phi_{i}^{n}}{\delta t}+\frac{1}{V_{i}} \sum_{f}\left[\mathbf{A} \cdot \mathbf{u}^{n}\right]_{f}[\phi]_{f}^{n}=S_{i}^{n}
$$

where $n$ indicates the $n^{\text {th }}$ time step, $V_{i}$ is the $i^{\text {th }}$ cell volume, and subscript $f$ indicates the cell (control volume) face.

In the fluid domain, the solid material will occupy the fluid volume. The partial-cell (PC) method is adopted to deal with this problem. The effective cell volume will be a fraction of the original cell volume:

$$
V_{\text {eff }}=\left(1-f_{\text {solid }}\right) V=\theta V
$$

where $V_{\text {eff }}$ is the effective cell volume, $f_{\text {solid }}$ is the solid volume fraction in each cell, $V$ is the original cell volume, and $\theta$ is the effective volume fraction.

\section{Moving-Solid Algorithm}

In order to deal with the moving of solid body, the PC method and a source function [30] are added to simulate the movement.

Consider the conservation of mass. A cell volume is $V$, and an obstacle volume and surface is $V_{o b s(t)}$ and $A_{o b s(t)}$, respectively.

The volume $V$ can be expressed:

$$
\oint_{A} u \cdot n d A=\frac{d V_{o b s(t)}}{d t}
$$

If the obstacle volume increases, the volume of the fluids decreases in a cell. In other words, $d V_{\text {obst }} / d t$ is greater than zero. The new continuity equation for the volume $V$ can be expressed as:

$$
\nabla \cdot u=\frac{1}{V} \frac{d V_{o b s(t)}}{d t}=\phi(x, y, z, t)
$$

where $\varphi=$ internal source function.

\section{Motion Analysis of Discrete Bodies}

To predict the movement of the solid body, DEM model is adopted. The multi-phase fluid force acting on the solid bodies and solid surfaces as shown in Fig. 1 are integrated to calculate the movement of the solid objects. To acquire the fluid force calculated from the computational fluid mechanics part, pressure sensing grids (see Fig. 2(a)) are patched on the block surfaces. The density of the sensing grid can be defined by the user according to the gradient of the pressure field the solid may admit during the motion analysis. An interpolation scheme as shown in Fig. 2(b) is used to find the weighting average pressure value at the sensing node of the block from the data of the fluid cells surrounding it.

Inverse square distance method is used in the interpolation part. A weighting of the fluid cell is showed in Eq. (11). Sum

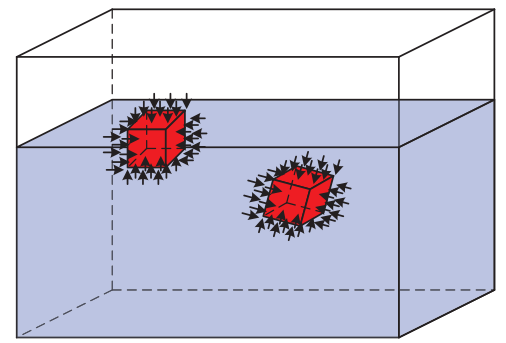

Fig. 1. Interaction motion analysis among discrete solid bodies and multiphase fluid.

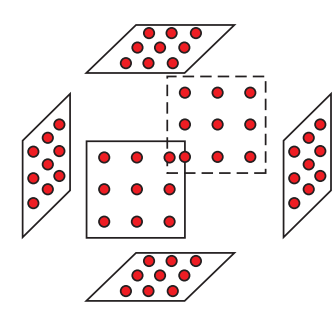

(a)

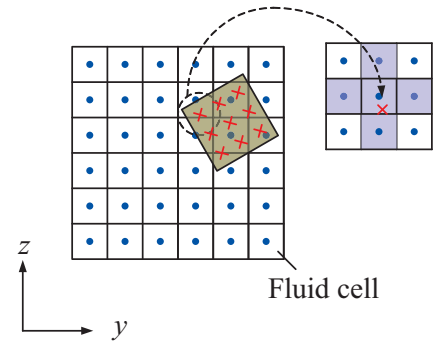

(b)
Fig. 2. (a) a $3 \times 3$ pressure integration grid on each surface of a cubic solid (b) a top view of the interpolation scheme of the surface pressure of a body at a sensing grid from the data of its surrounding fluid cells

up the weighted pressure of fluid cells, and we can get the pressure (Eq. (12)).

$$
\begin{gathered}
\omega_{i}=\frac{1}{d_{0 i}^{2}} / \sum_{i=1}^{N} \frac{1}{d_{0 i}^{2}} \\
P_{0}^{*}=\sum_{i=1}^{N} \omega_{i} P_{i}
\end{gathered}
$$

where $d_{o i}$ is the distance between numerical pressure point and the ambient fluid cells, $N$ is the total number of the weighting points. In three-dimension Cartesian grid, $N=7$. However, only the fluid cells $(\theta>0)$ will be included in the calculation.

In the present paper, rigid bodies are used to demonstrate the capability and accuracy of this two-way coupled movingsolid algorithm in fluid-solid interaction analysis. After finding the external forces exerted on the rigid bodies, an equivalent force-moment system (see Fig. 3) applied on the block centroid can be determined for the motion analysis of the block in the next time step.

As shown in Fig. 3, the resultant forces and moments applied on each block contain the effects from the fluid forces and the contact forces. Newton's equations (Eq. (13)) and Euler's equations (Eq. (14)) are applied to prescribe the motion of block of mass $\mathrm{m}$ in the spatial-temporal space.

$$
\sum F_{x}=m a_{x} ; \sum F_{y}=m a_{y} ; \sum F_{z}=m a_{z}
$$




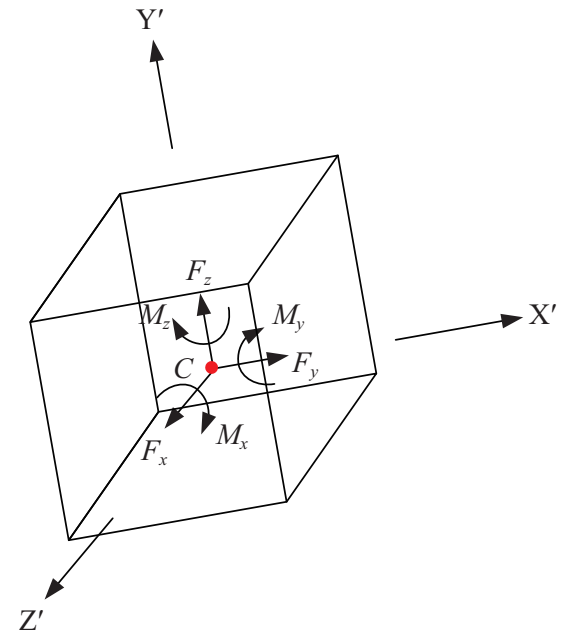

Fig. 3. Equivalent force-moment system for a body exert by the hydrodynamic forces.

$$
\begin{aligned}
& \sum M_{x^{\prime}}=I_{x^{\prime}} \alpha_{x^{\prime}}-\left(I_{y^{\prime}}-I_{z^{\prime}}\right) \omega_{x^{\prime}} \omega_{z^{\prime}} ; \\
& \sum M_{y^{\prime}}=I_{y^{\prime}} \alpha_{y^{\prime}}-\left(I_{z^{\prime}}-I_{x^{\prime}}\right) \omega_{z^{\prime}} \omega_{x^{\prime}} ; \\
& \sum M_{z^{\prime}}=I_{z^{\prime}} \alpha_{z^{\prime}}-\left(I_{x^{\prime}}-I_{y^{\prime}}\right) \omega_{x^{\prime}} \omega_{y^{\prime}}
\end{aligned}
$$

where $a_{i}$ is the $\mathrm{i}$-th component of the acceleration vector, the $\alpha_{i}$ is the i-th component of the angular acceleration vector, the $\omega_{i}$ is the $\mathrm{i}$-th component of the angular velocity vector, the subscripts $x, y, z$ are the global coordinate axes, and the subscripts $x^{\prime}, y^{\prime}, z^{\prime}$ are the principal axes of the mass moment of inertias $I x^{\prime}, I y^{\prime}, I z^{\prime}$.

Explicit central difference time integration scheme is used to solve these equations of motion. The velocity value at time $t+\frac{\Delta t}{2}$ can be obtained as:

$$
v_{i}^{\left(t+\frac{\Delta t}{2}\right)}=\left[\left(1-\frac{\alpha \Delta t}{2}\right) v_{i}^{\left(t-\frac{\Delta t}{2}\right)}+\left(\frac{F_{i}}{m}+g_{i}\right) \Delta t\right] \frac{1}{1+\frac{\alpha \Delta t}{2}}
$$

where $\alpha$ is the numerical damping and $g_{i}$ is the $\mathrm{i}$-th component of the gravity acceleration. Then, the component of the displacement increment within a time step $\Delta t$ is

$$
\Delta x_{i}=v_{i}^{\left(t+\frac{\Delta t}{2}\right)} \Delta t
$$

If the resultant force does not pass through the centroid of the block, a rotating moment will exist. Since the Euler's equation expressed by Eq. (14) is in the local coordinate system $\left(x^{\prime}, y^{\prime}, z^{\prime}\right)$ for the simplicity in analysis. Therefore, we have to transform the external moment expressed in global coordinate system into the current local coordinate system $\left(x^{\prime}\right.$, $\left.y^{\prime}, z^{\prime}\right)$ by Eq. (17).

$$
\begin{aligned}
& {\left[\begin{array}{l}
M_{x^{\prime}} \\
M_{y^{\prime}} \\
M_{z^{\prime}}
\end{array}\right]=[\mathbf{A}]\left[\begin{array}{l}
M_{x} \\
M_{y} \\
M_{z}
\end{array}\right],[\mathbf{A}]=\left[\begin{array}{lll}
a_{1} & a_{2} & a_{3} \\
b_{1} & b_{2} & b_{3} \\
c_{1} & c_{2} & c_{3}
\end{array}\right]} \\
& a_{1}=\cos \left(x^{\prime}, x\right), a_{2}=\cos \left(x^{\prime}, y\right), a_{3}=\cos \left(x^{\prime}, z\right) \\
& b_{1}=\cos \left(y^{\prime}, x\right), b_{2}=\cos \left(y^{\prime}, y\right), b_{3}=\cos \left(y^{\prime}, z\right) \\
& c_{1}=\cos \left(z^{\prime}, x\right), c_{2}=\cos \left(z^{\prime}, y\right), c_{3}=\cos \left(z^{\prime}, z\right)
\end{aligned}
$$

Similar to the analysis of displacement vector, the angular velocity vector $\omega^{\prime}$ at time $t+\frac{\Delta t}{2}$ calculated by the central difference scheme can be calculated as follows:

$\omega_{z^{\prime}}^{\left(t+\frac{\Delta t}{2}\right)}=\left[\left(1-\frac{\beta \Delta t}{2}\right) \omega_{z^{\prime}}^{\left(t-\frac{\Delta t}{2}\right)}+\left(\frac{M_{z^{\prime}}^{(t)}}{I_{z}^{\prime}}+\frac{\left(I_{x^{\prime}}-I_{y^{\prime}}\right)}{I_{z^{\prime}}}\right) \omega_{x^{\prime}} \omega_{y^{\prime}} \Delta t\right] \frac{1}{1+\frac{\beta \Delta t}{2}}$

where $\beta$ is the damping ratio of rotation. This angular velocity vector is further inversely transformed back to the global coordinate system by Eq. (17). Finally, the increment of rotation within this time step is determined as:

$$
\Delta \theta_{i}=\omega_{i}^{t+\frac{\Delta t}{2}} \Delta t
$$

Those two displacement increment vector and the rotation increment vector are used to update the new position of each block in the computational space for the next step analysis.

\section{SIMULATION RESULT}

In this part, two cases are discussed by using the numerical method. The first case is tsunami waves by landslide. The result will be compared and validated with the laboratory data provided from references [16]. The other case is a floating body in still water. This simulation shows the interaction between water waves and solid motion.

\section{Landslide Tsunami Simulation}

The results of the 3D subaerial and submerged landslide simulations will be presented in this section. The simulations cover different physical parameters with a wide range of initial landslide elevation $(\delta)$ and specific weight.

The side view of the 3D landslide setup is shown in Fig. 4. An 1:2 slope is placed in a $7.4 \mathrm{~m}$ wide tank. The initial water depth is $3.0 \mathrm{~m}$. A wedge with the size of $45.5 \times 61.0 \times 91.0 \mathrm{~cm}$ is sliding down along the slope into the water. The numerical domain is generated with uniform grid size of $\Delta x=7.5 \mathrm{~cm}$, $\Delta z=3.75 \mathrm{~cm}$ and nonuniform in y direction with $\Delta y_{\min }=0.6$ $\mathrm{cm}$ near the free-surface. The density of water is $1000.0 \mathrm{~kg} / \mathrm{m}^{3}$. The air density is set to $0.0 \mathrm{~kg} / \mathrm{m}^{3}$ and is excluded from the 
Table 1. Laboratory setup in the OSU experiments.

\begin{tabular}{|c|c|c|}
\hline Run number & $\delta(\mathrm{cm})$ & Density $\left(\mathrm{kg} / \mathrm{m}^{3}\right)$ \\
\hline 13 & 45.4 & 2144.0 \\
\hline 18 & 5 & 2144.0 \\
\hline 24 & -5 & 2144.0 \\
\hline 39 & 45.4 & 2791.0 \\
\hline 34 & 5 & 2791.0 \\
\hline 31 & -5 & 2791.0 \\
\hline 40 & 45.4 & 3425.0 \\
\hline 44 & 5 & 3425.0 \\
\hline 48 & -5 & 3425.0 \\
\hline
\end{tabular}

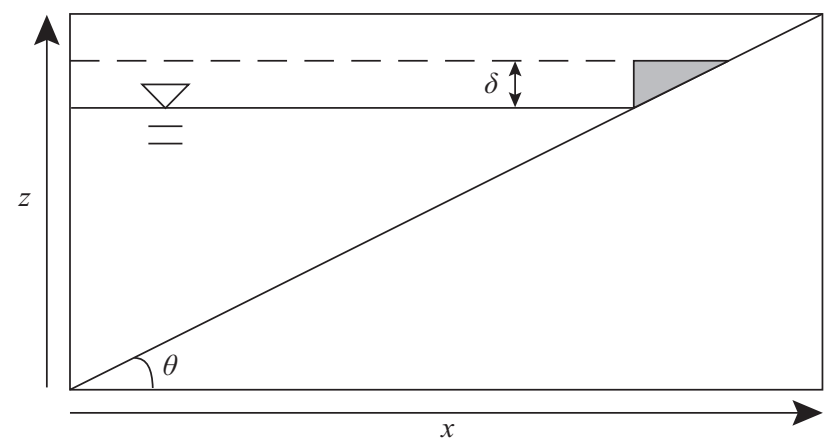

Fig. 4. The side view of the centerline cross section of landslide setup. An 1:2 slope is placed in a tank with length $(x)$ is $7.4 \mathrm{~m}$ and height $(z)$ is $3.7 \mathrm{~m}$. A wedge with the size of $45.5 \times 61.0 \times 91.0 \mathrm{~cm}$ is placed on the slope. The distant between initial wedge top and still water level is delta $(\delta) . \theta$ is slope angle.

simulation. Table. 1 shows the laboratory setup in the experiments. With difference initial elevations of the wedge ( $\delta$ and density), the numerical results are compared with laboratory data. The detail wedge parameters can be found in Table 1 .

In the laboratory setup, the motion of the wedge is controlled by wheels and rails under it. In order to control the wedge motion in the numerical model, the displacements in the $\mathrm{x}$ and $\mathrm{z}$ directions are projected on to the slope. The rotation of the wedge is set to zero to satisfy the experiment setup. The wedge motion is then reduced from 6 DOF (degrees of freedom) to $1 \mathrm{DOF}$.

A friction forcing term is added in the momentum equation and is determined by trial and error. The friction force between the wedge and the slope is assumed to be a linear function of the net weight of the wedge in the water:

$$
f_{k}=\mu_{k} N_{e f f}
$$

where $f_{k}$ is the kinetic friction force, $\mu_{k}$ is the coefficient of kinetic friction and $N_{\text {eff }}$ is the force normal to the slope.

Considering the effect of buoyancy, the effective normal force, $N_{\text {eff }}$ normal to the slope can be described as:

$$
N_{\text {eff }}=N-B=\left(\rho_{\text {solid }}-\rho_{\text {water }}\right) V g \cos \theta
$$

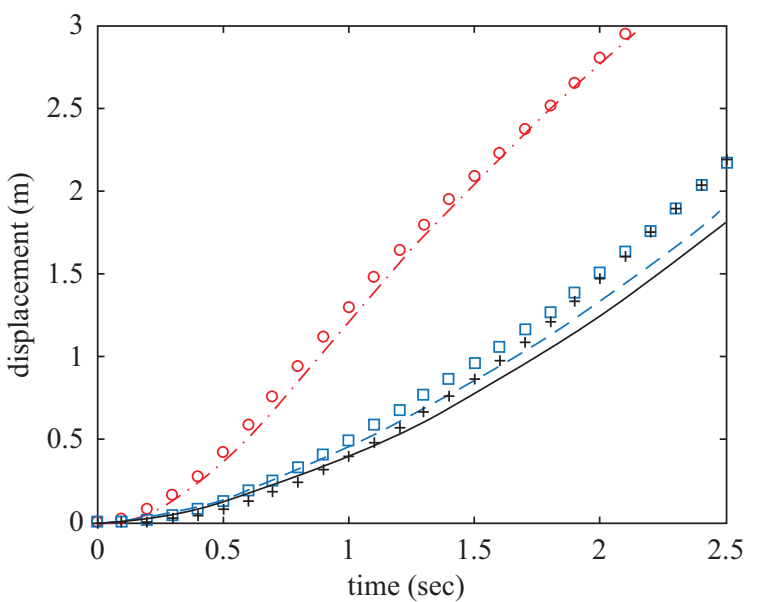

Fig. 5. The time-history trajectories of the landslide wedge. Symbol $\circ$, $\square$ and + denote experimental data of run13, run18 and run24, respectively. The corresponding lines are the numerical results. The density of the wedge is $2144.0 \mathrm{~kg} / \mathrm{m}^{3}$.

where $N$ is normal force, $B$ is buoyancy, $\rho_{\text {solid }}$ is the density of wedge, $\rho_{\text {water }}$ is the density of water, $V$ is the volume of wedge, $g$ is gravity and $\theta$ is slope angle.

By summing up the pressure force on the cube faces, the normal force $N_{\text {eff }}$ can be obtained. The coefficient of kinetic friction is nearly a constant, 0.1 , in all the simulations. The constant friction coefficient shows that the simulation results are independent from the empirical friction coefficient, and the results can be served as the model validations.

Figs. 5 7 show the comparisons of wedge trajectories obtained from the laboratory experiments and the numerical simulation. Fig. 5 is the trajectories with wedge density equals to $2144.0 \mathrm{~kg} / \mathrm{m}^{3}$. The red dash dot line is the trajectory of the subarial wedge (run13). The blue dash line (run18) and the solid black line (run24) are trajectories of submerged wedges.

In run13, the trajectory predicted by the coupled model shows good agreement with experimental data. From $0 \mathrm{sec}$ to $0.9 \mathrm{sec}$, the wedge speeds up by gravity. After moving into the water surface, the speed of the wedge is reduced by water resistance. The wedge velocity then slows down from $0.9 \mathrm{sec}$ to $1.5 \mathrm{sec}$. After time $=1.5 \mathrm{sec}$, the wedge velocity reaches a terminal velocity and the trajectory displays in a straight line. In run 18 and run24, there is no de-acceleration of the moving wedges because there is no wedge penetration from the air to the water. Fig. 5 also shows that with the same wedge density, the higher the initial wedge elevation, the faster the wedge speeds.

Fig. 6 and Fig. 7 respectively shows the trajectories with wedge density equals to $2791.0 \mathrm{~kg} / \mathrm{m}^{3}$ and $3425.0 \mathrm{~kg} / \mathrm{m}^{3}$. Comparing with the greater density, both the friction force and water resistance are smaller. The motion of wedge reaches terminal velocity quickly. Comparing with run13, run39 and run40, the initial landslide elevations $(\delta)$ are the same and the difference between those cases is wedge density. It's showed a positive correlation between density and velocity. 


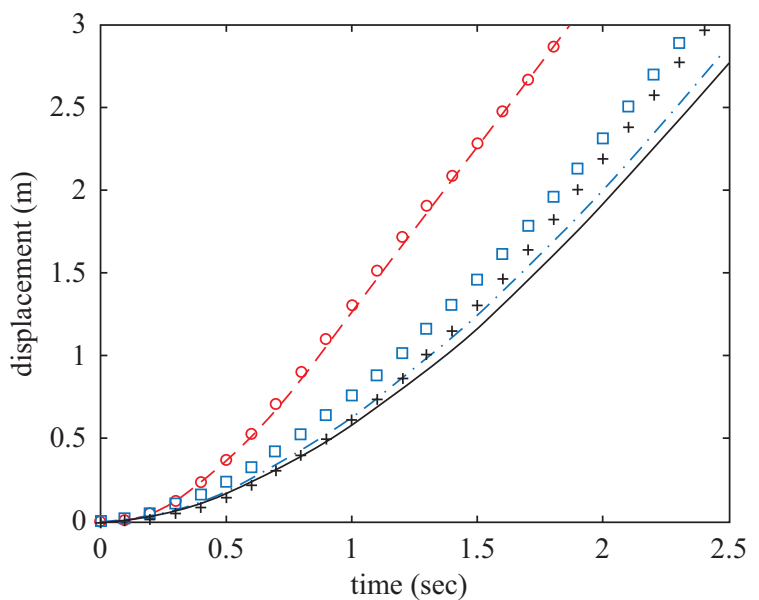

Fig. 6. The time-history trajectories of the landslide wedge. Symbol $\circ$, $\square$ and + denote experimental data of run39, run34 and run31. The corresponding lines are the numerical results. The density of the wedge is $2791.0 \mathrm{~kg} / \mathrm{m}^{3}$.

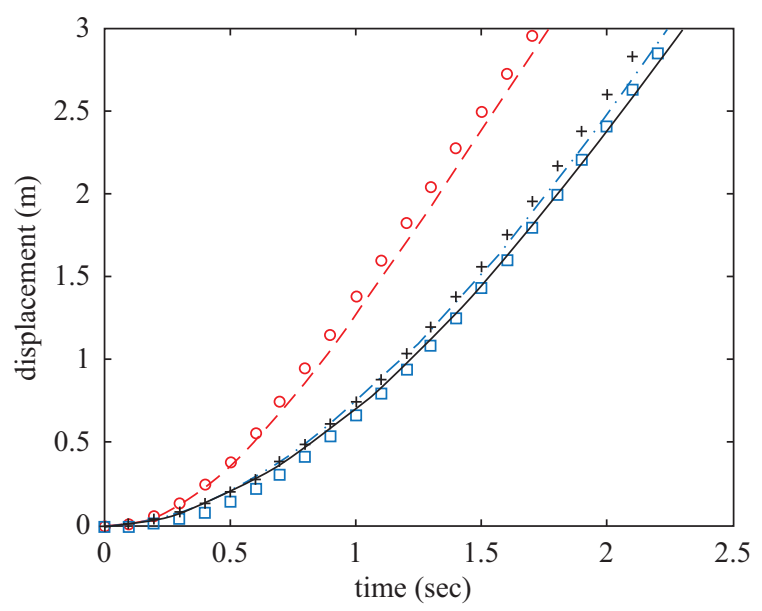

Fig. 7. The time-history trajectories of the landslide wedge. Symbol $\circ$, and + denote experimental data of run 40, run44 and run48. The corresponding lines are the numerical results. The density of the wedge is $3425.0 \mathrm{~kg} / \mathrm{m}^{3}$.

\section{Floating Cube Simulation}

We further extend the DOF from 1 to 6 by simulating a cube floating on the water surface. The movement of the cube is determined by the DEM which integrates the fluid pressure acting on the cube surface. The specific trajectory is specified. All the movements are predicted via the coupled model. The density of the cube varies from $600 \mathrm{~kg} / \mathrm{m}^{3}$ to $800 \mathrm{~kg} / \mathrm{m}^{3}$ to demonstrate the force balance among the solid cube and fluids.

The uniform Cartesian grid system is employed in the simulation with grid size $\Delta x=0.476 \mathrm{~cm}, \Delta y=0.476 \mathrm{~cm}$ and $\Delta z=$ $0.476 \mathrm{~cm}$. A cube is placed in the tank. The length of tank is $10 \mathrm{~cm}$ in each direction and cube is $5 \times 5 \times 5 \mathrm{~cm}$. The density of water is $1000 \mathrm{~kg} / \mathrm{m}^{3}$. The density of air is $0 \mathrm{~kg} / \mathrm{m}^{3}$. The still water level is located at $\mathrm{z}=6 \mathrm{~cm}$ while in the bottom the computational domain locates at $\mathrm{z}_{\mathrm{bottom}}=0 \mathrm{~cm}$. All the boundaries are no-slip boundary except for the zero pressure at the ceiling $\left(\mathrm{z}_{\text {top }}=10 \mathrm{~cm}\right)$. The cube center is same as the still water level (at $\mathrm{z}=6 \mathrm{~cm}$ ). Three different densities of the cube are simulated: $\rho=600 \mathrm{~kg} / \mathrm{m}^{3}, 700 \mathrm{~kg} / \mathrm{m}^{3}$ and $800 \mathrm{~kg} / \mathrm{m}^{3}$ (Figs. 8 10). From the simulation results, the interaction between wave pressure, gravity, and buoyancy can be observed.

The numerical results of $\mathrm{x}-\mathrm{z}$ plane at $\mathrm{y}=5 \mathrm{~cm}$ with different densities are presented in Figs. 8, 9, and 10. At time $=0.05 \mathrm{sec}$, the displacements of cubes are close to zero. At time $=0.25$ sec, the cubes are affected by gravity and water pressure which causes the displacement of cube. Fig. 8 shows the angle view of the free-surface profiles and velocity vectors in which cube density is $600 \mathrm{~kg} / \mathrm{m}^{3}$. At the time $=0.25 \mathrm{sec}$, cube moves downward and the water velocity pointed towards the top of the cube. Fig. 9 and Fig. 10 show the lager velocity vector field with greater densities 700 and $800 \mathrm{~kg} / \mathrm{m}^{3}$, respectively. In Fig. 10, the cube moving speed is also greater than others. At $0.25 \mathrm{sec}$, the wedge is downward and water around the cube moves towarding the top face of cube.

Time-history displacements of the cube center locations are shown in Fig. 11. From time $=0 \mathrm{sec}$ to $0.2 \mathrm{sec}$, all the cubes move downward. Cubes with larger density move faster. From time $=0.2 \mathrm{sec}$ to $0.4 \mathrm{sec}$, because the pressure force is increasing and it makes the cube moving up. Fig. 11 also shows that the denser cube has longer moving period and larger moving amplitude. All the moving trajectories show a decaying trend. The decaying trend is caused by the water waves generated by the oscillating cubes. The water waves transfer the cube energy to the fluid. The cubes moves up and down in the fluid after $t=1.0 \mathrm{sec}$. The averaged locations of cube centers are located at $\mathrm{z}=5.5,5.0$, and $4.5 \mathrm{~cm}$ with density $=$ $600 \mathrm{~kg} / \mathrm{m}^{3}, 700 \mathrm{~kg} / \mathrm{m}^{3}$ and $800 \mathrm{~kg} / \mathrm{m}^{3}$ respectively. All three values are close to theoretical values for cubes in the calm water.

\section{CONCLUSION}

In this study, we developed a two-way coupled movingsolid method which is able to predict both the fluid and solid motions. The model is validated with laboratory data of the landslide generated waves. Good results can be seen. By coupling with DEM model, the moving solid method is able to simulate both fluid and solid motion at the same computational cycle. The trajectories of landside tsunami agree with the experimental data. It also shows a positive correlation between velocity and density, velocity and delta $(\delta)$. The cases of floating cube with different cube density are simulated for demonstrating the performance of two-way coupled moving-solid algorithm in 6 DOF. The results show that the floating cubes move up and down in the water and generate waves. The oscillations of the cubes have mean elevations which are close to the theatrical cube location in the calm water.

However, the validation of the landslide wedge is $1 \mathrm{DOF}$ only, we shall move on to the floating-cube simulations for the 6 DOF validation with the experimental data. The current 


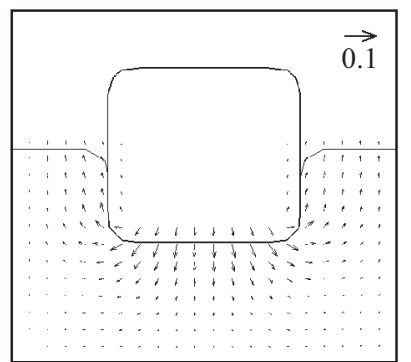

$\mathrm{T}=0.05$

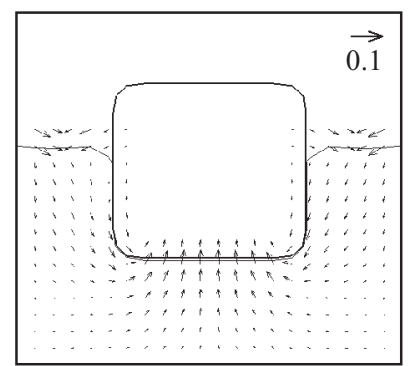

$\mathrm{T}=0.25$

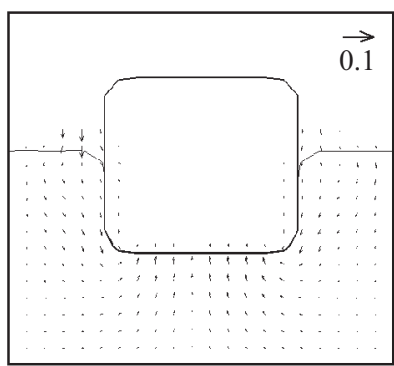

$\mathrm{T}=2.0$

Fig. 8. The snapshots of cube and free-surface locations as well as the velocity vectors of $x-z$ plane at $y=5 \mathrm{~cm}$ with cube density $=600 \mathrm{~kg} / \mathrm{m}^{3}$.

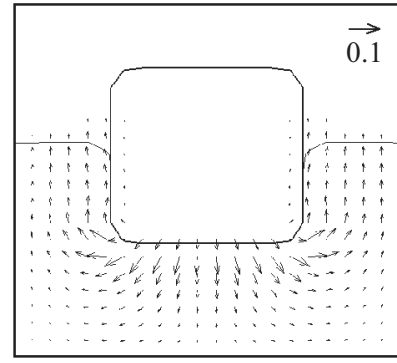

$\mathrm{T}=0.05$

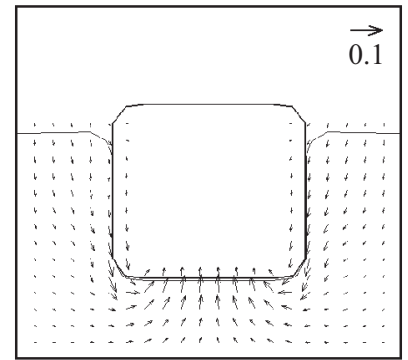

$\mathrm{T}=0.25$

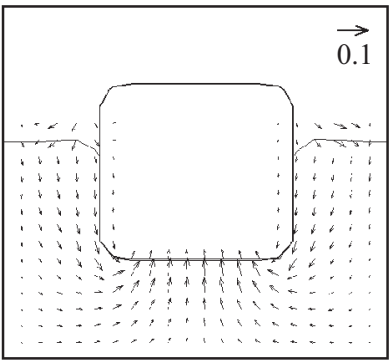

$\mathrm{T}=2.0$

Fig. 9. The snapshots of cube and free-surface locations as well as the velocity vectors of $x-z$ plane at $y=5 \mathrm{~cm} \mathrm{with} \mathrm{cube} \mathrm{density}=700 \mathrm{~kg} / \mathrm{m}^{3}$.

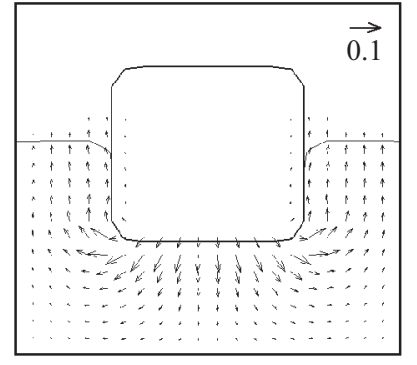

$\mathrm{T}=0.05$

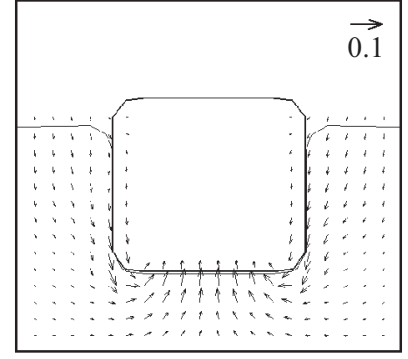

$\mathrm{T}=0.25$

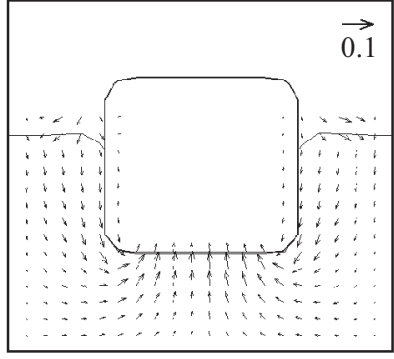

$\mathrm{T}=2.0$

Fig. 10. The snapshots of cube and free-surface locations as well as the velocity vectors of $x-z$ plane at $y=5 \mathrm{~cm}$ with cube density $=800 \mathrm{~kg} / \mathrm{m}^{3}$.

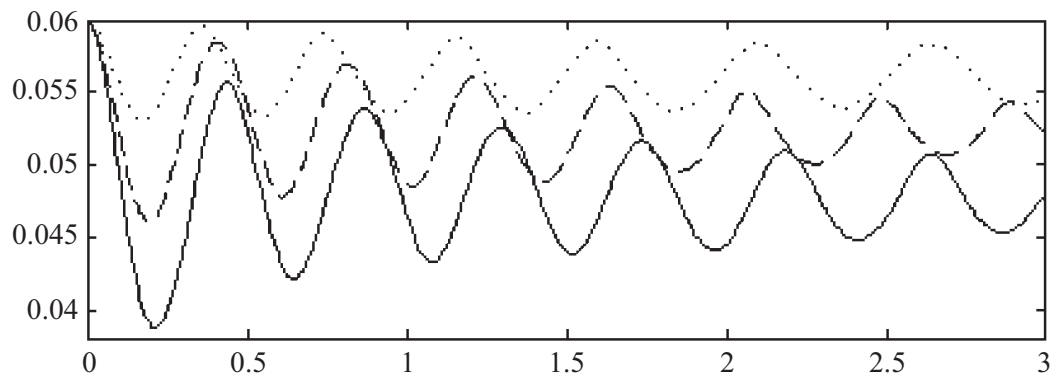

Fig. 11. Time histories of the cube center vertical displacements. Dot line denotes cube density $\rho=600 \mathrm{~kg} / \mathrm{m}^{3}$, dashed line denotes $\rho=700 \mathrm{~kg} / \mathrm{m}^{3}$, and solid line denotes $\rho=800 \mathrm{~kg} / \mathrm{m}^{3}$.

model can only be executed on a single CPU which limits the computation domain and simulation speed. The parallel version will be presented in the near feature. As for the future works, the pressure interpolation scheme in the current model can also be improved to have better accuracy on the solid surface. 


\section{ACKNOWLEDGMENTS}

The authors are grateful to National Science Council, Taiwan, for the financial support under the Grant NSC 95-2116M-008-006-MY3, with TEC Contribution Number 00040.

\section{REFERENCES}

1. Attaway, S. W., Hendrickson, B. A., Plimpton, S. J., Gardner, D. R., Vaughan, C. T., Brown, K. H., and Heinstein, M. W., "A parallel contact detection algorithm for transient solid dynamics simulations using PRONTO3D," Computational Mechanics, Vol. 22, pp. 43-159 (1998).

2. Balaras, E., "Modeling complex boundaries using an external force field on fixed Cartesian grids in large-eddy simulations," Computers and Fluids, Vol. 33, pp. 375-404 (2004).

3. Bost, C., Cottet, G. H., and Maitre, E., "Convergence analysis of a penalization method for the three-dimensional motion of a rigid body in an incompressible viscous fluid," SIAM Journal on Numerical Analysis, Vol. 48, pp. 1313-1337 (2010)

4. Cundall, P. A., "Formulation of a three-dimensional distinct element model-Part II: Mechanical calculations for motion and interaction of a system composed of many polyhedral blocks," International Journal of Rock Mechanics and Mining Science \& Geomechanics Abstracts, Vol. 25, pp. 117-125 (1988).

5. Cundall, P. A., "Formulation of three-dimensional distinct element modelPart I: A scheme to detect and represent contacts in a system composed of many polyhedral blocks," International Journal of Rock Mechanics and Mining Science \& Geomechanics Abstracts, Vol. 25, pp. 107-116 (1988).

6. Fadlun, E. A., Verzicco, R., Orlandi, P., and Mohd-Yusof, J., "Combined immersed-boundary finite-difference methods for three-dimensional complex flow simulations," Journal of Computational Physics, Vol. 161, pp. 35-60 (2000).

7. Glowinski, R., Pan, T. W., and Periaux, J., "A fictitious domain method for Dirichlet problem and applications," Computer Methods in Applied Mechanics and Engineering, Vol. 111, pp. 283-303 (1994).

8. Glowinski, R., Pan, T. W., and Periaux, J., "Distributed Lagrange multiplier methods for incompressible viscous flow around moving rigid bodies," Computer Methods in Applied Mechanics and Engineering, Vol. 151, pp. 181-194 (1998).

9. Heinrich, P., "Nonlinear water waves generated by submarine and aerial landslides," Journal of Waterway, Port, Coastal, and Ocean Engineering, Vol. 118, pp. 249-266 (1992)

10. Hirt, C. W. and Nicholes, B. D., "Volume of fluid (VOF) method for the dynamics of free boundaries," Journal of Computational Physics, Vol. 39, pp. 201-225 (1981).

11. Hu, H. H., Patankar, N. A., and Zhu, M. Y., "Direct numerical simulations of fluid-solid systems using the arbitrary Lagrangian-Eulerian technique," Journal of Computational Physics, Vol. 169, pp. 427-462 (2001).

12. Jia, W. and Nakamura, Y., "Incompressible flow solver of arbitrarily moving bodies with rigid surface," JSME International Journal, Vol. 39, pp. 315-325 (1996).

13. Kothe, D. B. and Mjolsness, R. C., "RIPPLE: a new model for incompressible flows with free surfaces," AIAA Journal, Vol. 30, pp. 2694-2700 (1992)

14. Lin, P., "A multiple-layer $\sigma$-coordinate model for simulation of wavestructure interaction," Computers and Fluids, Vol. 35, pp. 147-167 (2006).

15. Lin, P. and Li, C. W., "A $\sigma$-coordinate three-dimensional numerical model for surface wave propagation," International Journal for Numerical Methods in Fluids, Vol. 38, pp. 1045-1068 (2002).

16. Liu, P. L.-F., Wu, T.-R., Raichlen, F., Synolakis, C., and Borrero, J., "Runup and rundown from three-dimensional sliding masses," Journal of Fluid Mechanics, Vol. 536, pp. 107-144 (2005).

17. Mittal, R. and Iaccarino, G., "Immersed boundary methods," Annual Review of Fluid Mechanics, Vol. 37, pp. 239-261 (2005).

18. Mohd-Yusof, J., "Combined immersed boundaries/B-splines methods for simulations of flows in complex geometries," CTR Annual Research Briefs, Center for Turbulence Research, Stanford University, pp. 317-327 (1997).

19. Müller, M., Charypar, D., and Gross, M., "Particle-based fluid simulation for interactive applications," Proceedings of the 2003 ACM SIGGRAPH/ Eurographics Symposium on Computer Animation, San Diego, California, pp. 154-159 (2003).

20. Müller, M., Solenthaler, B., Keiser, R., and Gross, M., "Particle-based fluid-fluid interaction," Proceedings of the 2005 ACM SIGGRAPH/ Eurographics Symposium on Computer Animation, Los Angeles, California, pp. 29-31 (2005).

21. Peskin, C. S., "Flow patterns around heart valves: a numerical method," Journal of Computational Physics, Vol. 10, pp. 252-271 (1972).

22. Randrianarivelo, N., Pianet, G., Vincent, S., and Caltagirone, J. P., "Numerical modelling of solid particle motion using a new penalty method," International Journal for Numerical Methods in Fluids, Vol. 47, pp. 12451251 (2005).

23. Shen, L. W. and Chan, E. S., "Numerical simulation of fluid-structure interaction using a combined volume of fluid and immersed boundary method," Ocean Engineering, Vol. 35, pp. 939-952 (2008).

24. Shi, G. H., "Manifold method of material analysis," Transactions of the Ninth Army Conference on Applied mathematics and Computing, Minneapolis, Minnesota, USA, pp. 51-76 (1992).

25. Shi, G. H., "Manifold method," Proceedings of the First International Forum on Discontinuous Deformation Analysis (DDA) and Simulations of Discontinuous Media, California, USA, pp. 52-104 (1996).

26. Shi, G. H., Discontinuous Deformation Analysis - A New Numerical Model for the Statics and Dynamics of Block System, Ph. D. Dissertation, Department of Civil Engineering, University of California, Berkeley (1988).

27. Ting, E. C., Shih, C., and Wang, Y. K., "Fundamentals of a vector form intrinsic finite element: Part I. basic procedure and a plane frame element," Journal of Mechanics, Vol. 20, pp. 113-122 (2004).

28. Ting, E. C., Shih, C., and Wang, Y. K., "Fundamentals of a vector form intrinsic finite element: Part II. plane solid elements," Journal of Mechanics, Vol. 20, No. 2, pp. 123-132 (2004).

29. Wang, C. Y., Sheng, J., Huang, C. J., and Chen, M. H., "Motion analysis of mixed polyhedral and ellipsoidal particles," Proceedings of 6th International Conference on Computation of Shell and Spatial Structures, Cornell University, Ithaca, NY, USA, pp. 28-31 (2008).

30. Wu, T.-R., A Numerical Study of Three-Dimensional Breaking Waves and Turbulence Effects, Ph.D. Dissertation, Department of Civil Engineering, Cornell University, New York (2004).

31. Zhao, D., Nezami, E. G., Hashash, Y. M. A., and Ghaboussi, J., "Threedimensional discrete element simulation for granular materials," International Journal for Computer-Aided Engineering and Software, Vol. 23, pp. 749-770 (2006).

32. Zhong, Z. H. and Nilsson, L., "A contact searching algorithm for general 3-D contact-impact problems," Computers and Structures, Vol. 34, pp. $327-$ 335 (1990). 